\title{
Multivariate Extreme Value Theory - A Tutorial with Applications to Hydrology and Meteorology
}

\begin{abstract}
In this paper, we provide a tutorial on multivariate extreme value methods which allows to estimate the risk associated with rare events occurring jointly. We draw particular attention to issues related to extremal dependence and we insist on the asymptotic independence feature. We apply the multivariate extreme value theory on two data sets related to hydrology and meteorology: first, the joint flooding of two rivers, which puts at risk the facilities lying downstream the confluence; then the joint occurrence of high speed wind and low air temperatures, which might affect overhead lines.
\end{abstract}

Keywords: Multivariate extreme value theory, Joint extreme hazards, Asymptotic independence

MSC: 62G32, 62H05, 62H12

DOI 10.2478/demo-2014-0003

Received June 2, 2013; accepted April 24, 2014.

\section{Introduction}

In many fields, we are interested in modeling extreme hazards in order to estimate the risk that a particular community or industrial structure incurs. For example, in hydrology ([3]), the ultimate interest in flood frequency analysis is the estimation of the T-year flood discharge which is the level exceeded every $T$ years on average; in meteorology $([4,5,8,35])$, we are interested in the analysis of daily fastest-mile wind speeds or in daily maximum temperatures; in insurance applications, the estimation of the upper tail of the claim size distribution is of major interest in order to determine the net premium of a reinsurance contract; in finance, the Value-At-Risk of a portofolio is one of the most important risk measures that have been used by investors or fund managers in an attempt to assess or predict the impact of unfavourable events; in geology and seismic analysis, applications can be found in the magnitudes of earthquakes and the associated losses.

The univariate extreme value theory is now relatively standard to model the tail of the distribution of a scalar random variable: it provides an asymptotic justification for the generalized Pareto distribution to be an appropriate model for the distributoin of excesses over a suitably chosen high threshold $([1,10])$.

In many situations, we need to take into account the link between several rare events, since the effects might be compounded if they occur simultaneously. Accurate assesments of the probabilities of joint extreme events are sought in diversity of applications from environmental impact assessment $([9,11,12,14,18,32,33$, $38,39,47])$ to financial risk management $([20,30,36,42])$ and Internet traffic modelling $([31,37])$.

The multivariate extreme value theory and methods concern the characterization, estimation and extrapolation of the joint tails of multidimensional distributions.

\footnotetext{
*Corresponding Author: Anne Dutfoy: EDF R\&D, 1, avenue du Général de Gaulle, 92141 CLAMART Cedex, France, E-mail: anne.dutfoy@edf.fr

Sylvie Parey: EDF R\& D, 6 quai Watier, 78401 Chatou Cedex, France, E-mail: sylvie.parey@edf.fr Nicolas Roche: EDF R\& D, 6 quai Watier, 78401 Chatou Cedex, France, E-mail: nicolas.roche@edf.fr
} 
In this paper, we provide a tutorial on multivariate extreme value methods. We draw particular attention to issues related to extremal dependence, as it is a critical issue in many applications to model correctly extreme risks. Then, we analyse two data sets related to hydrology and meteorology, in the light of some multivariate extreme techniques: the hydrology application investigates the case where the joint flooding of two rivers puts a risk at common facilities lying downstream the confluence; the meteorology application deals with risks on overhead lines due to high speed wind jointly with low air temperatures.

The paper is organized as follows: in section 2, the basics of the multivariate extreme value theory are reminded. We give general results in dimension $d \geq 2$ but we focus on the bivariate framework as most of the multivariate applications are bivariate. Section 3 is devoted to asymptotic independence within the bivariate framework and to some statistical methods for its estimation and modeling. Both applications are respectively studied in Section 4 and Section 5. We conclude in Section 6.

\section{Basics of the multivariate extreme value distributions}

This section is devoted to the main results of the multivariate extreme value theory and presents some methods for multivariate inference. It also explains the specificities of the multivariate framework compared to the univariate one. We conclude by giving some limits of the use of multivariate extreme value distributions which might lead to dramatic consequences. More details can be found in $[1,10,15,19,20,22,28]$.

\subsection{What makes the multivariate context so specific}

In the univariate context, the theory is based on the manipulation of a few concepts that were found very useful in analysing extreme values. However, in the multivariate context, these notions lose their evident definition. We have to reconsider them in order to set an adapted signification.

For example, the study of multivariate extremes raises issues of ordering, as there are many ways to order multivariate vectors depending on whether we consider one component or all of them. Marginal ordering is not a total ordering: it is not always possible to compare two vectors. Besides, the component-wise maximum of observations might not correspond to one observed data, which makes any approach based on the component-wise maximum delicate to handle.

In relation with the latter issue, the definition of what makes a multivariate observation extreme is rather tricky: is it enough that just a single coordinate attains an exceptional value, or should it be extreme in all dimensions simultaneously?

A fundamentally new issue arising in the multivariate context is that of dependence, which describes how margninal extremes interact.

\subsection{Key results}

Similar to the univariate case, the first historical multivariate extreme value method is based on block maxima: observations are partitioned into blocks, each block is reduced to its vector of component-wise maxima. Then the collection of block maxima is modeled as an independent sample of a multivariate extreme value distribution.

The multivariate block maxima approach is based on the Theorem 2.1. Let $\left(\boldsymbol{X}^{1}, \ldots, \boldsymbol{X}^{n}\right)$ be a sequence of independent random vectors of dimension $d \geq 2$, identically distributed according to the distribution function $F$ and $\boldsymbol{M}_{n}=\boldsymbol{X}^{1} \vee \ldots \vee \boldsymbol{X}^{n}$ their component-wise maximum.

Theorem 2.1 (Independent and Identically Distributed Case). $F$ is in the domain of attraction of a multivriate extreme value distribution $G$ (noted $F \in \mathcal{D}(G)$ ) if there exist sequences of vectors $\left(\boldsymbol{a}_{n}\right)_{n}>\mathbf{0}$ and $\left(\boldsymbol{b}_{n}\right)_{n}$ such that $\boldsymbol{a}_{n}^{-1}\left(\boldsymbol{M}_{n}-\boldsymbol{b}_{n}\right)$ converges in distribution to a non-degenerate d-variate distribution function $G$ with 
non-degenerate margins such that:

$$
\mathbb{P}\left(\boldsymbol{a}_{n}^{-1}\left(\boldsymbol{M}_{n}-\boldsymbol{b}_{n}\right) \leq \boldsymbol{x}\right)=F^{n}\left(\boldsymbol{a}_{n} \boldsymbol{x}+\boldsymbol{b}_{n}\right) \longrightarrow G(\boldsymbol{x}), \quad n \rightarrow+\infty
$$

Thanks to Sklar's Theorem ([34, Theorem 2.10.9]) any multivariate distribution function $F$ writes as the composition of a copula $C_{F}$ and its marginal distributions $F_{i}$ as follows:

$$
F\left(x_{1}, \ldots, x_{d}\right)=C_{F}\left(F_{1}\left(x_{1}\right), \ldots, F_{d}\left(x_{d}\right)\right)
$$

In the multivariate extreme value distribution, the dependence structure is written when the margins have been reducted to standard Frechet distributions rather than uniform ones. Although the choice of marginal distributions essentially makes no difference, some properties are most naturally seen for this particular choice, which motivates some particular inference strategies.

Then $G$ and its copula $C_{G}$ write, in terms of the margins $G_{i}$ and the stable tail dependence function $\ell: \mathbb{R}^{d} \longrightarrow$ $\mathbb{R}$ :

$$
\begin{gathered}
G(\boldsymbol{x})=\exp \left\{-\ell\left(-\log \left[G_{1}\left(x_{1}\right)\right], \ldots,-\log \left[G_{d}\left(x_{d}\right)\right]\right)\right\} \\
C_{G}(\boldsymbol{u})=\exp \left\{-\ell\left(-\log \left(u_{1}\right), \ldots,-\log \left(u_{d}\right)\right)\right\}
\end{gathered}
$$

$\ell$ has the following properties: $l(s \cdot)=s l(\cdot)$ for $0<s<\infty ; l\left(\boldsymbol{e}_{j}\right)=1$ for $j=1, \ldots, d$, where $\boldsymbol{e}_{j}$ is the $j$ th unit vector in $\mathbb{R}^{d} ; v_{1} \vee \cdots \vee v_{d} \leq l(\boldsymbol{v}) \leq v_{1}+\cdots+v_{d}$ for $\boldsymbol{v} \in[\mathbf{0}, \infty) ; l$ is convex.

Considering relation (1) for each margin $i=1, \ldots, d$, we obtain:

$$
F_{i}^{n}\left(a_{n, i} x_{i}+b_{n, i}\right) \longrightarrow G_{i}\left(x_{i}\right), \quad n \longrightarrow \infty
$$

Therefore, each $G_{i}$ is by itself a univariate extreme value distribution function and $F_{i} \in \mathcal{D}\left(G_{i}\right)$. $G_{i}$ writes:

$$
G_{i}\left(x_{i}\right)=\exp \left\{-\left[1+\xi_{i}\left(\frac{x_{i}-\mu_{i}}{\sigma_{i}}\right)\right]^{-1 / \xi_{i}}\right\}
$$

defined on $\left\{x_{i} \mid 1+\xi_{i}\left(x_{i}-\mu_{i}\right) / \sigma_{i}>0\right\}$, where $-\infty<\mu_{i}<\infty, \sigma_{i}>0$ and $-\infty<\xi_{i}<\infty$.

Proposition 2.2. $F \in \mathcal{D}(G)$, with $C_{G}$ the copula of $G$, if and only if it satisfies (4) together with:

$$
\lim _{t \rightarrow \infty} C_{F}^{t}\left(u_{1}^{1 / t}, \ldots, u_{d}^{1 / t}\right)=C_{G}(\boldsymbol{u}), \quad \forall \boldsymbol{u} \in[0,1]^{d}
$$

If $F \in \mathcal{D}(G)$, the tail of the distribution $F$ writes:

$$
F(\boldsymbol{x}) \simeq \exp \left\{-\ell\left(-\log \left[F_{1}\left(x_{1}\right)\right], \ldots,-\log \left[F_{d}\left(x_{d}\right)\right]\right)\right\}
$$

for $x_{j}$ such that $F_{j}\left(x_{i}\right) \simeq 1, \forall j$, where the tail of the margins $F_{i}$ are modeled thanks to a generalized Pareto distribution, which writes:

$$
F_{i}\left(x_{i}\right)=1-\lambda_{i}\left\{1+\xi_{i}\left(\frac{x_{i}-u_{i}}{\sigma_{i}}\right)\right\}^{-1 / \xi_{i}} \quad x_{i}>u_{i}
$$

for large $u_{i} . \xi_{i}$ is the tail index of $F_{i}$ and is the same as for the extreme value distribution $G_{i}$.

\subsection{Some tail dependence functions}

We note that $G$ is an infinite dimensional object which does not have a finite parametrization: it is indexed by the function $\ell$, which makes $G$ difficult to handle. A quite popular approach is to use some well chosen parametric sub-models. We give here a brief overview of the most popular models for the tail dependence function $\ell$ : they have been shown in various case studies to be particularly successful in combining analytical tractability with practical applicability. [1] gives a more exhaustive list. 
- Bivariate logistic model introduced by Gumbel (1960) ([24]):

$$
\ell\left(v_{1}, v_{2}\right)=\left(v_{1}^{1 / \alpha}+v_{2}^{1 / \alpha}\right)^{\alpha}, \quad \forall v_{i}>0
$$

with parameter $0<\alpha \leq 1 . \alpha$ measures the strength of dependence between the two coordinates. In particular, independence and complete dependence corresponds to $\alpha=1$ and $\alpha \downarrow 0$.

- Bivariate asymetric logistic model introduced by Tawn (1988) ([43]):

$$
\ell\left(v_{1}, v_{2}\right)=\left(1-\psi_{1}\right) v_{1}+\left(1-\psi_{2}\right) v_{2}+\left\{\left(\psi_{1} v_{1}\right)^{1 / \alpha}+\left(\psi_{2} v_{2}\right)^{1 / \alpha}\right\}^{\alpha}, \quad \forall v_{i}>0
$$

with parameters $0<\alpha \leq 1$ and $0 \leq \psi_{i} \leq 1$.

It is an asymetric extension of the bivariate Gumbel model. Independence arises for $\alpha=1$ and $\psi_{1}=\psi_{2}=0$; for $\psi_{1}=\psi_{2}$, we obtain a mixture of independence and the logistic model.

- Multivariate extensions introduced by Tawn (1990) ([44]):

$$
\ell(\boldsymbol{v})=\sum_{c \in C_{d}}\left\{\sum_{j \in c}\left(\psi_{c, j} v_{j}\right)^{1 / \alpha_{c}}\right\}^{\alpha_{c}}, \quad \forall v_{j}>0
$$

where $C_{d}$ is the collection of non-empty subsets $c$ of $\{1, \ldots, d\}, 0<\alpha_{c} \leq 1, \psi_{c, j} \geq 0$ and $\sum_{j \in c} \psi_{c, j}=1$ for all $j=1, \ldots, d$. A simple special case is:

$$
\ell(\boldsymbol{v})=\left(v_{1}^{1 / \alpha}+\cdots+v_{d}^{1 / \alpha}\right)^{\alpha}, \quad \forall v_{i}>0
$$

Genest et Rivest ([23]) characterize the copulas corresponding to (12) as the only extreme value copulas that are also Archimedean copulas.

Tawn ([44]) also mentions the following trivariate extension:

$$
\ell(\boldsymbol{v})=\left\{\left(v_{1}^{1 / \alpha}+v_{2}^{1 / \alpha}\right)^{\alpha / \beta}+v_{3}^{1 / \beta}\right\}^{\beta}, \quad \forall v_{j}>0
$$

where $0<\alpha \leq \beta \leq 1$, featuring bivariate symmetric logistic dependence with parameter $\alpha$ for the first two coordinates and with parameter $\beta$ for the other pairs of coordinates.

In the bivariate case, the dependence information $\ell$ may be written in terms of Pickands dependence function $A$ defined as:

$$
A(t)=\ell(1-t, t)
$$

where $t \in[0,1] . A$ is convex and satisfies $(1-t) \vee t \leq A(t) \leq 1, t \in[0,1]$.

The case $A(t)=1$ features the independence and the case $A(t)=(1-t) \vee t$ features the complete dependence. More precisely, given the convexity of $A(t)$ and the bound constraints, the exact independence is equivalent to $A(1 / 2)=1$ and the complete dependence is equivalent to $A(1 / 2)=1 / 2$.

Conversely, $\ell$ writes:

$$
\ell\left(v_{1}, v_{2}\right)=\left(v_{1}+v_{2}\right) A\left(\frac{v_{2}}{v_{1}+v_{2}}\right)
$$

Using (2), (3), (7) and (14), we get:

$$
\begin{gathered}
G\left(x_{1}, x_{2}\right)=\exp \left\{\log \left[G_{1}\left(x_{1}\right) G_{2}\left(x_{2}\right)\right] A\left(\frac{\log \left[G_{2}\left(x_{2}\right)\right]}{\log \left[G_{1}\left(x_{1}\right) G_{2}\left(x_{2}\right)\right]}\right)\right\} \\
C_{G}\left(u_{1}, u_{2}\right)=\exp \left\{\log \left(u_{1} u_{2}\right) A\left(\frac{\log \left(u_{2}\right)}{\log \left(u_{1} u_{2}\right)}\right)\right\}, \\
F\left(x_{1}, x_{2}\right)=\exp \left\{\log \left[F_{1}\left(x_{1}\right) F_{2}\left(x_{2}\right)\right] A\left(\frac{\log \left[F_{2}\left(x_{2}\right)\right]}{\log \left[F_{1}\left(x_{1}\right) F_{2}\left(x_{2}\right)\right]}\right)\right\}
\end{gathered}
$$

where the approximation of $F$ is valid for large $x_{1}, x_{2}$. 


\subsection{Multivariate Inference}

Statistical inference on multivariate observations needs to make a certain number of choices.

The first one concerns the approach that will be used: the block maxima approach, based on the analysis of the limiting distribution of component-wise maxima (1) or the Peak Over Threshold (POT) approach that directly estimates the joint tail distribution (7).

A common critique to block maxima methods, in the univariate as well as in the multivariate context, is that they throw away many relevant observations. Furthermore, they evaluate the joint distribution function of extreme values occurring within a block whose granularity might not be refined enough for the analysist.

On the contrary, POT methods employ all observations for which at least one coordinate exceeds a corresponding high threshold, using the censored maximum likelihood method where the likelihood of an observation depends on which of its components exceed the corresponding thresholds coordinates. For example, in the bivariate case, the likelihood contribution $\mathcal{L}\left(x_{1}, x_{2}\right)$ of the observation $\left(x_{1}, x_{2}\right)$ writes:

$$
L\left(x_{1}, x_{2}\right) \propto \begin{cases}F\left(u_{1}, u_{2}\right) & \text { if } x_{1} \leq u_{1}, x_{2} \leq u_{2} \\ \frac{\partial F}{\partial x_{1}}\left(x_{1}, u_{2}\right) & \text { if } x_{1}>u_{1}, x_{2} \leq u_{2} \\ \frac{\partial F}{\partial x_{2}}\left(u_{1}, x_{2}\right) & \text { if } x_{1} \leq u_{1}, x_{2}>u_{2} \\ \frac{\partial^{2} F}{\partial x_{1} \partial x_{2}}\left(x_{1}, x_{2}\right) & \text { if } x_{1}>u_{1}, x_{2}>u_{2}\end{cases}
$$

The second choice concerns the strategy to infer on the margins and the dependence structure seperately or simultaneously.

As a matter of fact, the multivariate inference can split into inference on the margins and then inference on the joint dependence structure $\ell$. According to the approach, observations are marginally fitted to either a univariate extreme value distribution or a generalized Pareto distribution. Then, the estimates of the margins are used to map the original data into approximate standard Frechet margins. These transformed data are supposed to follow the multivariate extreme value distribution (2) or the joint tail model (7) whose stable function $\ell$ remains to be estimated. Of course, one must have confidence in one's modeling of the margins, as it conditions the transformed data upon which the dependence structure is fitted.

Alternatively, it is possible to handle the full model as a whole. In this strategy, the margins and the dependence structure are modeled at the same time. It has the benefit of allowing some transfer of information between the margins and the dependence structure. However, the estimation of the whole model might be complex.

Another issue concerns the choice between a semi-parametric approach or a fully parametric one. Usually, extreme value methods tend to take advantage of the parametric form of the margins while the dependence structure $\ell$ can be done parametrically or non-parametrically.

Under a semi-parametric specification, a number of non-parametric estimators exist for the dependence structure. In the bivariate framework, many authors have proposed non parametric estimators of the Pickands function $A(t)([6,7,16,25,48])$. [1] compares the statistical properties of these non-parametric estimators: in general, these estimators have difficulties to respect the bounds and convexity constraints of $A(t)$.

Under a fully parametric specification, a particular model for the dependence structure $\ell$ is fitted to the data, which facilitates the inference step but increases the risk of mismodeling the tail of the joint distribution.

\section{Asymptotic independence}

This section focuses on asymptotic independence which appears as the main limit for the use of extreme value distributions to model the tail of a multivariate distribution: indeed, it limits the options for the extremal dependence structure to either asymptotic dependence or exact dependence. 
Berman (1961) ([2]) showed that asymptotic independence in a random vector of dimension $d>2$ with a regularly varying joint tail distribution is equivalent to pairwise asymptotic independence for all its component pairs. This result allows us to restrict the analysis to the bivariate case, so we confine the exposition to this setting.

\subsection{Definition}

To handle the dependence in the extreme values between the components of a bivariate random vector $\left(X_{1}, X_{2}\right)$, we introduce the coefficient of upper tail dependence $\chi$ that can be interpreted as the tendency for one variable to be extreme given that the other one is extreme.

Definition 3.1. The random variates $X_{1}$ and $X_{2}$, distributed according to the distribution functions $F_{1}$ and $F_{2}$, are asymptotically independent if and only if:

$$
\chi=\lim _{u \rightarrow 1^{-}} \mathbb{P}\left(F_{1}\left(X_{1}\right)>u \mid F_{2}\left(X_{2}\right)>u\right)=0
$$

The coefficient $\chi$ is a copula-based measure and can be expressed in terms of the copula $C_{F}$ of the bivariate distribution $F$ of $\left(X_{1}, X_{2}\right)$ :

$$
\chi=\lim _{u \rightarrow 1^{-}} \frac{1-2 u+C_{F}(u, u)}{1-u}
$$

In the case of asymptotic independence, the joint extremes occur with negligible probability compared to that of a single extreme. Conversely, if $\chi \neq 0$, the joint extremes occur with a probability comparable to that of a single extreme. $\chi$ is a measure for strength of dependence within the class of asymptotically dependent distributions. It increases with a rising degree of dependence at extreme levels.

The simplest example of asymptotic independence is the exact independence. But asymptotic independence also features a dependence at any quantile $u$ that gradually vanishes when $u \longrightarrow 1$. We can distinguish asymptotic independent data that exhibit some positive (resp. negative) association: observations for which both components exceed a large threshold occur more (resp. less) frequently than under exact independence.

\subsection{Limits of the multivariate extreme value distribution}

Within the class of multivariate extreme value distributions, it is possible to model asymptotic dependence using the appropriate dependence function $\ell$.

However, the only possible type of asymptotic independence is, in fact, the exact independence. We show it in the bivariate case, thanks to ([2]) (which corresponds to each pair $\left(X_{i}, X_{j}\right)$ with $i \neq j$ in the case of dimension $d>2$ ).

Defining the tail dependence function of the bivariate distribution function $F$ by:

$$
D_{F}\left(u_{1}, u_{2}\right)=1-C_{F}\left(1-u_{1}, 1-u_{2}\right)=\mathbb{P}\left(\cup_{j=1}^{j=2}\left\{F_{j}\left(X_{j}\right)>1-u_{j}\right\}\right)
$$

then $F \in \mathcal{D}(G)$ is equivalent to (see [1, pp 283]):

$$
\lim _{s \longrightarrow 0^{+}} s^{-1} D_{F}\left(s u_{1}, s u_{2}\right)=l\left(u_{1}, u_{2}\right)
$$

where $\ell$ is the stable dependence function asosciated with $G$. Considering (22) for $u_{1}=u_{2}=1$ and introducing Pickands function $A(t)=\ell(1-t, t)$, we get:

$$
\lim _{s \rightarrow 0} s^{-1}\left[1-C_{F}(1-s, 1-s)\right]=l(1,1)=2 A(1 / 2)
$$

Using (20), (23) leads to $\chi=2-2 A(1 / 2)$. We deduce that the only way to have $\chi=0$ is to impose $A(1 / 2)=1$, which is equivalent to the exact independence $A(t)=1$ and $C_{G}\left(u_{1}, u_{2}\right)=u_{1} u_{2}$. Using (17), it implies that $C_{F}\left(u_{1}, u_{2}\right) \simeq u_{1} u_{2}$ for $u_{i} \simeq 1$. 
In other words, if we note $p=\mathbb{P}\left(F_{1}\left(X_{1}\right)>1-1 / z, F_{2}\left(X_{2}\right)>1-1 / z\right)$ the probability of joint exceedances of the respective $1 / z$ tail quantiles, then asymptotic dependence writes $p=\mathcal{O}(1 / z)$ (since $\chi \neq 0$ ), whereas asymptotic independence writes $p=o(1 / z)$ (since $\chi=0)$. We showed that multivariate extreme value distributions only allow to model asymptotic independence through $p=\mathcal{O}\left(1 / z^{2}\right)$. Thus, they are not appropriate to model asymptotic independence for which $p=\mathcal{O}\left(1 / z^{\alpha}\right)$, with $1<\alpha$ and $\alpha \neq 2$. Asymptotic independence with positive association corresponds to $1<\alpha<2$ and with negative association, it corresponds to $\alpha>2$.

A wrong modelisation entails bad results which might cause damage: in the case of positive association, using a multivariate extreme value distribution with the exact independence structure to model the tail of the joint distribution leads to a probability $p$ lower than the real one; we have the inverse result in the case of negative association. Thus, before using the approximations (1) or (7), it is necessary to explore extremal dependence and test for asymptotic independence. If such is the case, one has to leave the framework of extreme value distributions and look for models describing the tails of the distributions in a more refined way. The reader may find different tail models in [3, 17, 29].

\subsection{Tests to detect asymptotic independence}

Literature on inference for asymptotic independence is large, e.g. in [27, 45, 46, 49]. More recently, [13] made a very exhaustive overview of the current state on testing asymptotically independent data. Among others, we can note the score-based tests, the gamma test, the madogram test.

The criteria for selecting the methods presented above are their simplicity and the fact that both applications presented hereafter base their analysis on them.

Curves $\chi(u)$ and $\bar{\chi}(u)$ : The measure $\chi$ can be rewritten in terms of the limit of a function of $C_{F}$ :

$$
\chi=\lim _{u \rightarrow 1^{-}} \chi(u), \quad \chi(u)=2-\frac{\log C_{F}(u, u)}{\log u}, \quad 0<u<1 .
$$

To measure extremal dependence under asymptotic independence, Coles introduced the measure:

$$
\begin{gathered}
\bar{\chi}=\lim _{u \rightarrow 1^{-}} \bar{\chi}(u), \\
\bar{\chi}(u)=\frac{2 \log \mathbb{P}\left[F_{1}\left(X_{1}\right)>u\right]}{\log \mathbb{P}\left[F_{1}\left(X_{1}\right)>u, F_{2}\left(X_{2}\right)>u\right]}-1=2 \frac{\log (1-u)}{\log \bar{C}_{F}(u, u)}-1
\end{gathered}
$$

where $\bar{C}_{F}(u, u)$ is the survival copula $\bar{C}_{F}\left(u_{1}, u_{2}\right)=1-u_{1}-u_{2}+C_{F}\left(u_{1}, u_{2}\right)$.

The couple of coefficients $(\chi, \bar{\chi})$ allows to discriminate between asymptotic dependence and asymptotic independence and, in the latter case, between positive or negative association: if $\bar{\chi}=1$ and $0<\chi \leq 1$, then the variables are asymptotically dependent and $\chi$ gives a measure of strength of the dependence; if $-1 \leq \bar{\chi}<1$ and $\chi=0$, then the variables are asymptotically independent and $\bar{\chi}$ gives a measure of strength of the associations.

The empirical versions of the functions $\chi(u)$ and $\bar{\chi}(u)$ provide some insight into the dependence structure of the variables at lower quantile levels and can be used as a means of model assessment. The sign of $\chi(u)$ determines whether the variables are positively or negatively associated at quantile level $u$.

Furthermore, in the particular case where $C$ is a bivariate extreme value copula with Pickands function $A$, then from (16), we get that $\chi(u)=2-2 A(1 / 2)$ for all $u$. It entails that identifying that the empirical version of $\chi(u)$ is constant for any $u \in[0,1]$ is a diagnostic for belonging to the bivariate extreme value class.

Standard Frechet space: One useful test to assess whether a mutivariate distribution is asymptotically dependent or independent consists in transforming the data into standard Frechet margins using the transformation $Z_{i}=-1 / \log F_{i}\left(X_{i}\right)$ and then in mapping the data from cartesian to pseudo-polar coordinates $(R, W)=\left(Z_{1}+Z_{2}, \frac{Z_{1}}{Z_{1}+Z_{2}}\right)$. The random variable $R$ gives a measure of distance from the origin and $W$ measures the angle on a $[0,1]$ scale. We note that $R \gg 1$ corresponds to the extreme data for which at least only one component is large. Among these data, $W \simeq 1$ or $W \simeq 0$ corresponds to those for wich only one component 
is extreme (respectively $Z_{1}$ or $Z_{2}$ ), while $W \simeq 0.5$ corresponds to the data for which both components are extreme.

Thus, the histogram of $W$ corresponding to the largest values of $R$ allows to distinguish independent variables for which the histogram comprises atoms of mass 0.5 on $W=0$ and $W=1$, from perfectly dependent variables for which the histogram comprises an unique atom of mass 1.0 at $W=0.5$. Inbetween histograms give an insight on the strength of dependence.

Ledford and Tawn test: Besides $(\chi, \bar{\chi})$, Ledford \& Tawn ([29]) introduce a third dependence coefficient, $\eta$, by assuming that the joint survivor function of $\left(Z_{1}, Z_{2}\right)$, where data have been mapped into standard Frechet margins, is a regularly varying function. Then $\eta$ is the tail index defined in (8) of the univariate variable $T=\min \left(Z_{1}, Z_{2}\right)$. Ledford and Tawn define a statistical test in order to distinguish asymptotic dependence from asymptotic independence and, in the latter case, the positive from the negative associations: asymptotic independence is equivalent to $\eta<1$. So they suggest to test for asymptotic independence by testing $\mathcal{H}_{0}=\{\eta=1\}$ against its alternative $\mathcal{H}_{1}=\{0<\eta<1\}$.

Falk and Michel : Falk and Michel ([21]) map the data into Weibull distribution margins, using $Z_{i}=$ $\log F_{i}\left(X_{i}\right)$. Then, they show that $Z_{1}$ and $Z_{2}$ are asymptotically independent if and only if $F_{c}(t)=\mathbb{P}\left(Z_{1}+Z_{2}<\right.$ $\left.c t \mid\left(Z_{1}+Z_{2}\right)<c\right)$ tends to $F(t)=t^{2}$ for all $t$ when $c \longrightarrow 0^{+}$. Alternatively, under asymptotic dependence, we have pointwise convergence of $F_{c}(t)$ towards $F(t)=t$ when $c \longrightarrow 0^{+}$.

\subsection{Models for asymptotic independence}

[13] gives an overview of the most recent models for asymptotically independent data. Hereafter, we focus on the Ledford and Tawn model ([29]) that accomodates both asymptotic dependence and asymptotic independence.

Transforming the initial data into standard Frechet margins, Ledford and Tawn assume that the joint survivor function of $\left(Z_{1}, Z_{2}\right)$ is a regularly varying function:

$$
\mathbb{P}\left[\left(Z_{1}>z, Z_{2}>z\right]=\mathcal{L}(z) z^{-1 / \eta} \quad \in[0,1]\right.
$$

where $\mathcal{L}$ is a slowly varying function: $\mathcal{L}(z)>0$ for $z>0$ high enough and $\forall x>0, \lim _{z \rightarrow+\infty} \mathcal{L}(x z) / \mathcal{L}(z)=1$. The rate of decay in (27) is largely controlled by $\eta$ (modulated by $\mathcal{L}$ ). Ledford and Tawn motivate their model considering a large number of examples, which are confirmed by [26]. [40] gives some counterexamples, however they seem somewhat pathological.

$\bar{C}_{F}$ and $\mathcal{L}$ are connected through $\bar{C}_{F}(u, u)=\mathcal{L}(-1 / \log u)(-\log u)^{1 / \eta}$, which leads to:

$$
\bar{\chi}=\lim _{u \rightarrow 1} \bar{\chi}(u)=2 \eta-1, \quad \chi=\lim _{z \rightarrow \infty} \mathcal{L}(z) z^{1-1 / \eta}
$$

Thus the Ledford and Tawn model enables to model:asymptotic dependence for $\eta=1$ if $\lim _{z \rightarrow \infty} \mathcal{L}(z)=c$ for $0<c \leq 1$; asymptotic independence with positive association for $1 / 2<\eta<1$ or for $\eta=1$ and $\lim _{z \rightarrow \infty} \mathcal{L}(z)=0$; near independence for $\eta=1 / 2$ and exact independence if in addition, $\mathcal{L}(z)=1$; asymptotic independence with negative association for $0<\eta<1 / 2$.

Examples of copulas $C_{F}$ obeying conditions imposed on $\mathcal{L}$ include any member of the Farlie-GumbelMorgenstein family of copulas:

$$
C_{F}\left(u_{1}, u_{2}\right)=u_{1} u_{2}\left[1+\alpha\left(1-u_{1}\right)(1-u 2)\right]
$$

with parameters $-1 \leq \alpha \leq 1$, for which $\chi(u)=2-\frac{\log \left[u^{2}\left\{1+\alpha(1-u)^{2}\right\}\right]}{\log u}$ and $\lim _{u \rightarrow 1^{-}} \chi(u)=0$; or the bivariate normal distribution with correlation $\rho$ which models asymptotic independence with positive association if $\rho>0$ and negative association if $\rho<0$ ([41]).

[17] presents a generalization of the model of Ledford and Tawn: they introduce a new parameter $\theta$ which describes the tail dependence. In some cases, this parameter can be related to $\eta$. 


\section{Example of joint flooding of rivers}

This section is devoted to one example illustrating the asymptotic dependence. It deals with the joint exceedance rate of two rivers. The estimation of the flooding hazard for facilities installed alongside of rivers is mainly based on univariate extreme value analysis of the observed flow series. However, in some cases, the installation lies juste upstream the confluence of two rivers and then can be affected by the backwash of the other river. Considering the floods of both rivers as independent in the risk analysis may lead to an underestimation of the risk.

Here, the multivariate extreme value theory is used to quantify the asymptotical dependence of two river flows in France and then the result is used to better estimate the risk associated with the compound flood events.

\subsection{Data analysis}

The observations are daily discharges simultaneously recorded on both rivers, during the years from 1958 to 2000 , in $m^{3} \cdot s^{-1}$.

Figure 1 plots the bidimensional observations in the physical space and in the rank space (using the empirical distribution function). It suggests a strong relationship between the discharges of both rivers at extreme levels, as we can distinguish an accumulation of data for which both components are extreme on figure $1(\mathrm{~b})$.

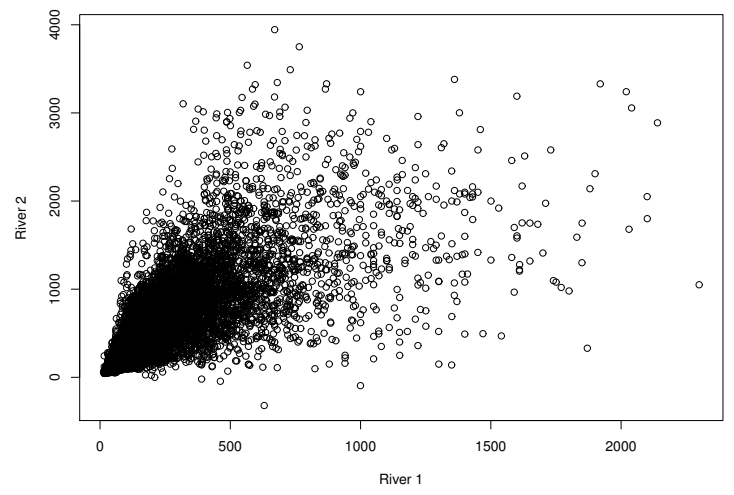

(a)

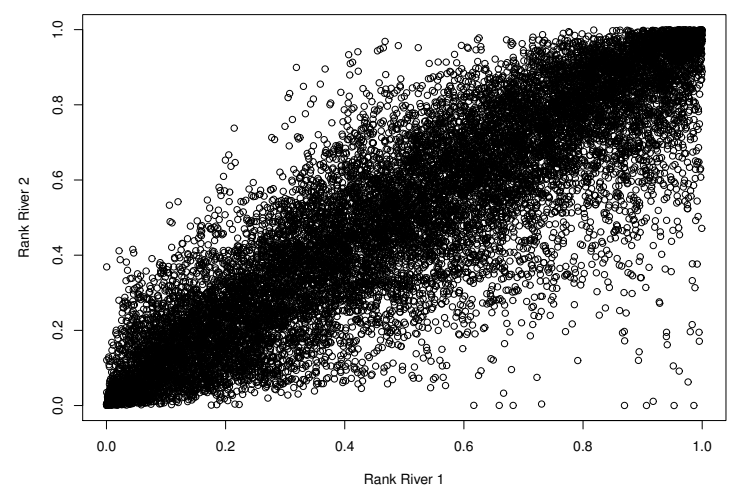

(b)

Fig. 1. Scatterplot of daily discharges of River 1 versus River 2 during the years from 01/01/1958 to 02/07/2000: (a) original data; (b) data mapped to uniform distribution.

To confirm it, figure 2 plots the histogram of $W$ for all the data for which at least one component exceeds its corresponding threshold: it corresponds to a model with a reasonably weak level of dependence, which is significantly different from independence.

Figure 3 plots the estimation of the tail coefficient $\eta$ of the $T$ variable defined within the Ledford \& Tawn test, with its 95\% point-wise confidence intervals: since all the confidence intervals contain the unit value, the hypothesis of asymptotic independence is not discarded.

Finally, figure 4 draws the empirical versions of the functions $\chi(u)$ and $\bar{\chi}(u)$ and confirms the asymptotic dependence. 


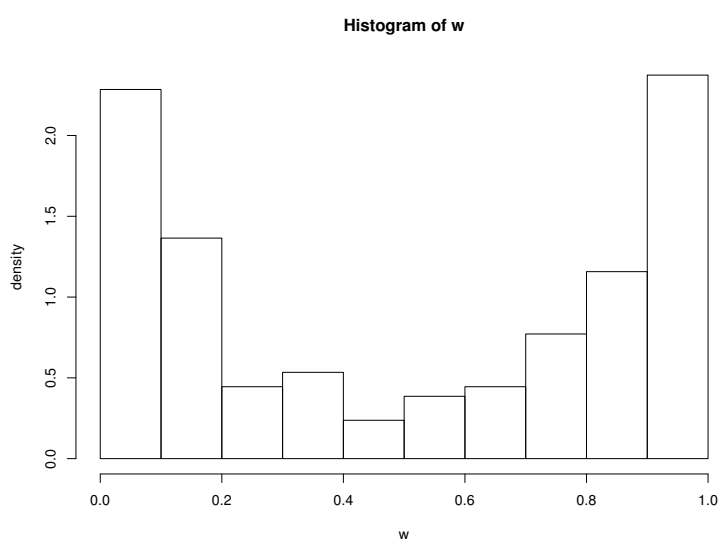

Fig. 2. Histogram of $W$ values in the point process analysis of both rivers' discharges.

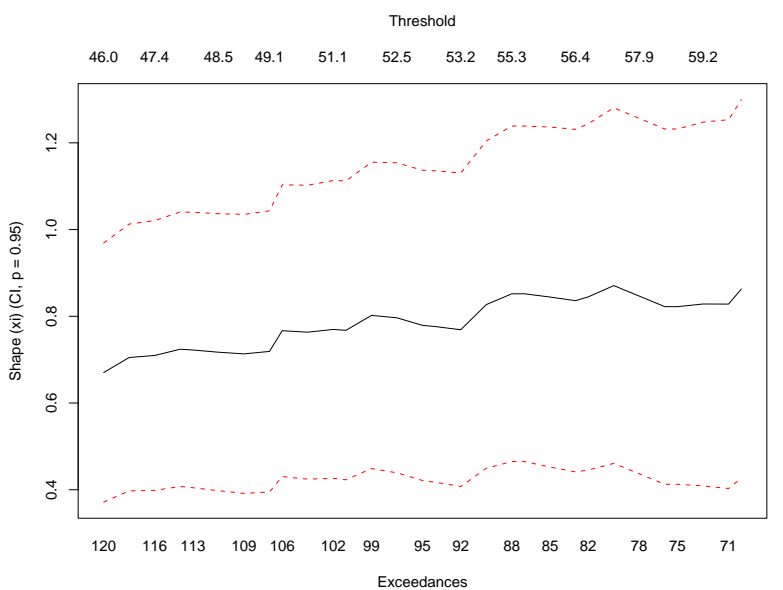

Fig. 3. Estimation of the coefficient of tail dependence $\eta$ of the variable $T=\min \left(Z_{1}, Z_{2}\right)$ for several exceedance thresholds.

\subsection{Joint tail modelisation}

The previous tests lend support to the use of asymptotically dependent models above sufficiently high thresholds. Thus, we model the tail of the distribution of joint discharges by an extreme value distribution as defined in (7) and (8).

To estimate the aproximate model, we fit the dependence structure with the logistic model. We perform both model fitting procedures presented in section 2.4 (the two-step approach and the all-at-once approach), using the censored maximum likelihood. They both lead to the same results summed up in table 1. Figures 5 and 6 are usual plots that enable us to appreciate the quality of the marginal fittings.

Figure 7 confirms the goodness of fit of the dependence model. As the dependence coefficient $\alpha$ is close to 1 , the strength of the asymptotic dependence is not so high.

\subsection{Model exploitation}

Once the model is fully estimated, it is possible to use it in order to get some original results.

For example, it gives access to the quantile curves at extreme levels that regroup all the combinations of the two rivers' discharges that correspond to the same risk level. Figure 8 draws the curves associated with the quantiles $(0.99,0.995,0.999)$. 


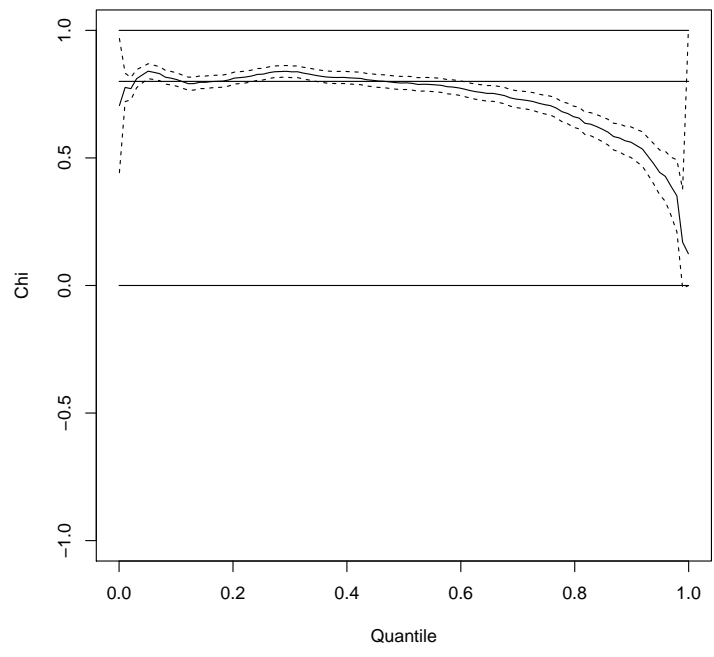

(a)

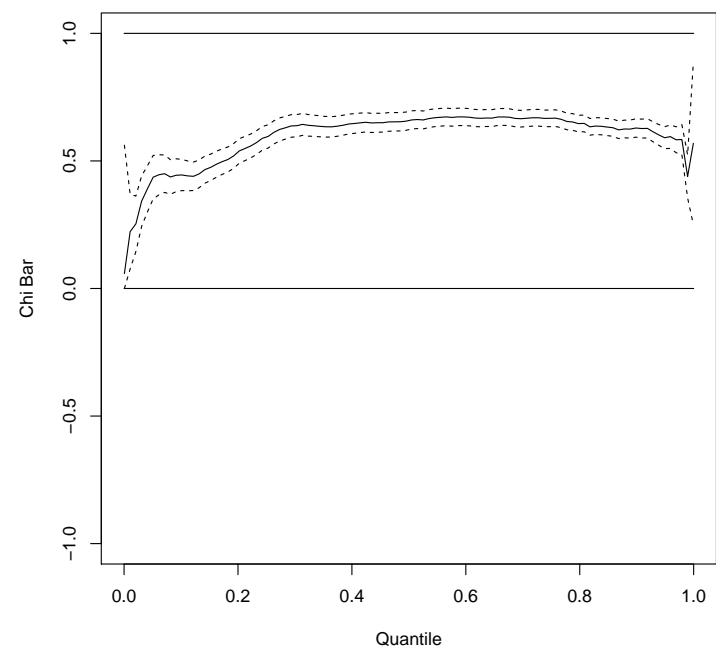

(b)

Fig. 4. Empirical version of the functions $u \rightarrow \chi(u)$ (a) et $u \rightarrow \bar{\chi}(u)$ (b) for the daily discharges of Rivers 1 and River 2 .

Table 1. Coefficients of the approximate model of the tail distribution, estimated by censored maximum likelihood.

\begin{tabular}{ccc}
\hline & River 1 & River 2 \\
\hline$u\left(m^{3} \cdot s^{-1}\right)$ & 1050 & 2150 \\
$\sigma\left(m^{3} \cdot s^{-1}\right)$ & 335.29 & 571.14 \\
$\xi$ & -0.126 & -0.259 \\
$\lambda$ & 0.012 & 0.0119 \\
\hline \multicolumn{3}{c}{ Dependence coeff. } \\
\hline$\alpha$ & 0.879 \\
\hline
\end{tabular}

One original and very useful result consists in fixing the discharge of one river to an extreme level and then studying the probabilistic distribution of the discharge of the other river. For example, when the discharge of the second river is fixed to its 100 -year return level $X_{2}^{100}=3898 \mathrm{~m}^{3} \cdot \mathrm{s}^{-1}$, we can evaluate the probabilistic features of the discharge of the first river: figure 9 draws the probability density function of the first river and table 2 gives its probabilistic features. Thus, when one river is in spate, it is possible to estimate the likely discharge of the second river and then take that level into account in safety studies.

Table 2. Probabilistic features of the River 1 discharges given that the second river discharge is fixed to its 100-year return level.

\begin{tabular}{lc}
\hline & River $1\left(m^{3} \cdot s^{-1}\right)$ \\
\hline Mean & 2513 \\
Standard deviation & 141 \\
Quantile 0.5 & 2517 \\
Quantile 0.25 & 2436 \\
Quantile 0.75 & 2629 \\
\hline
\end{tabular}



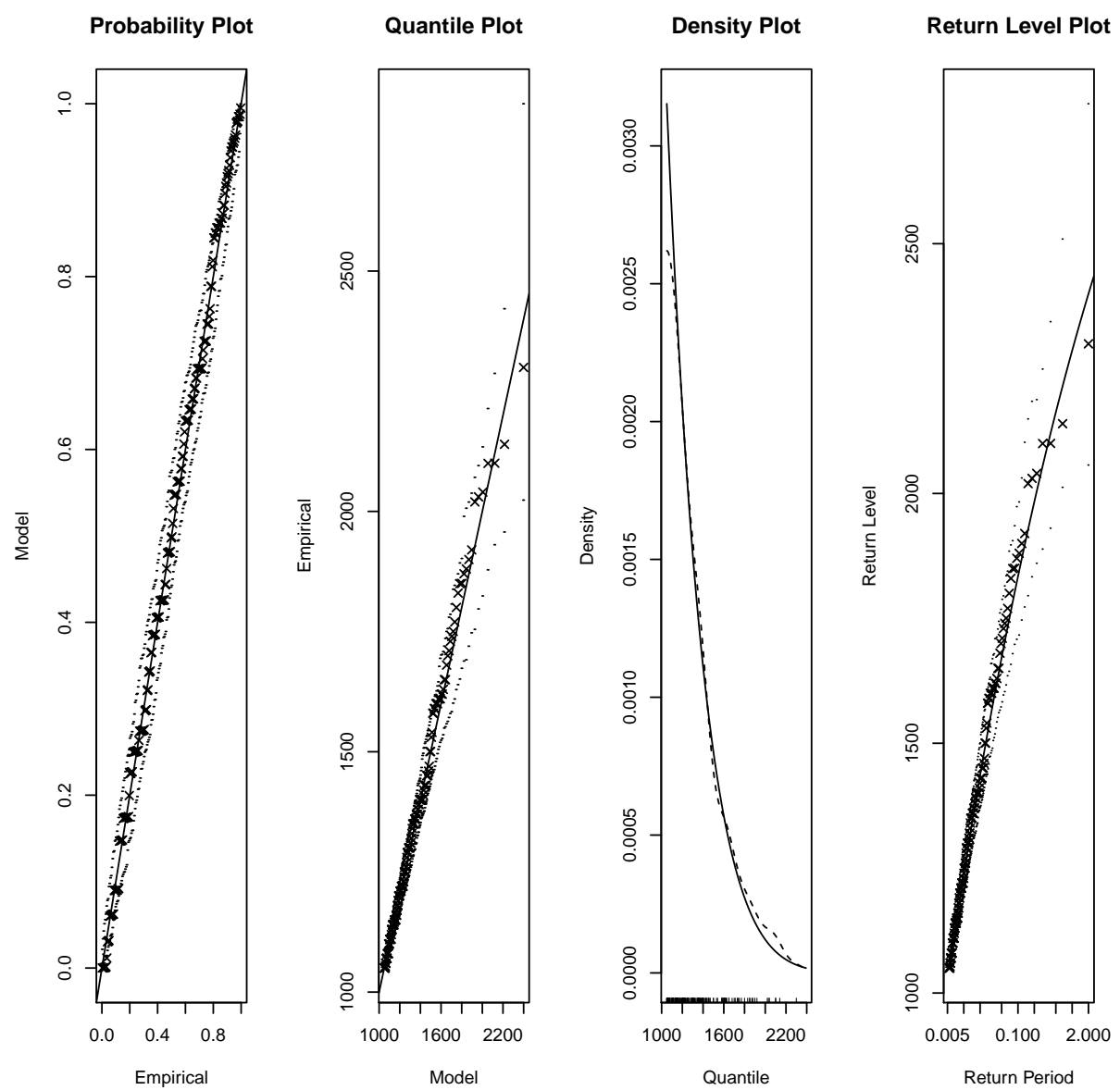

Fig. 5. Goodness of fit of the marginal model for River 1: probability plot, quantile plot, density plot and return level plot.

\section{Example relating to temperatures and wind speed hazards}

This section is devoted to a second example illustrating the asymptotic independence with negative association. It deals with the joint occurrence of high wind speed and low air temperatures at the same place, which might induce a risk on overhead lines: these lines might be overloaded with rime if temperature is very low, making the lines and poles more vulnerable to strong winds. Here again, the multivariate extreme value theory will help quantify the asymptotical dependence of both events for a given location in the centre of France.

\subsection{Data analysis}

The observations were recorded daily and simultaneously during the years from 1971 to 2011 . We recorded the daily maximal temperature in degrees Celsius and the maximal wind speed in $m \cdot \mathrm{s}^{-1}$.

In this case, we face an additional difficulty: temperature data cannot be considered as realizations of independent and identically random vectors as we can distinguish some seasonality plotted in figure 10. Furthermore, we had to take into consideration serial dependence between two consecutive measures. That's why we restricted our analysis to observations recorded during the extended winters (defined from November until March) and we also declusterized the series. 

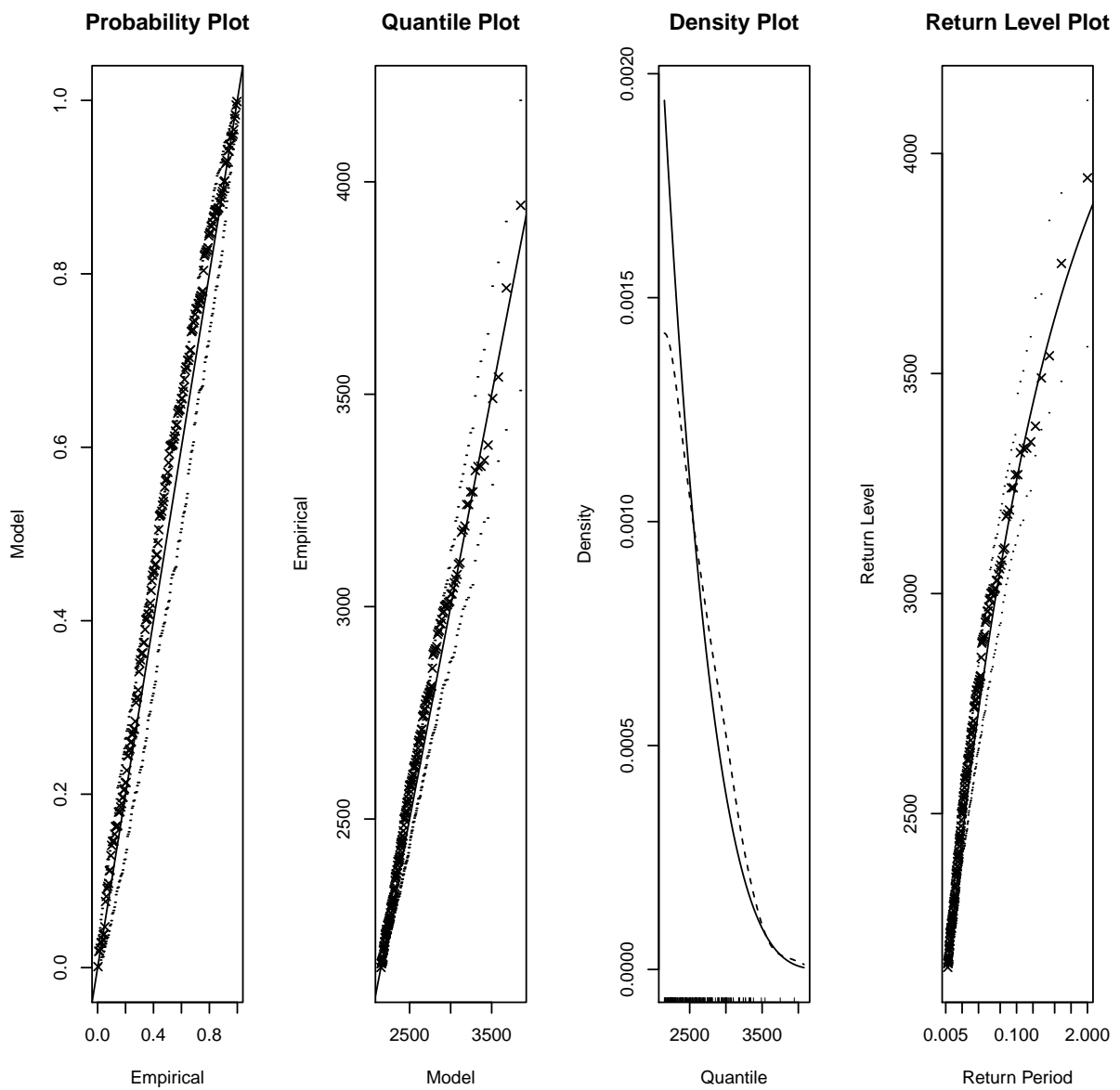

Fig. 6. Goodness of fit of the marginal model for River 2: probability plot, quantile plot, density plot and return level plot.

On the other hand, the wind speed data, restricted to the winters, could be considered as a stationary sequence with no serial dependence.

As we are interested in low values of temperature, we transform the temperature data into their negated values: thus, we study high extreme values of those new variables, which allows us to use the usual statistical tools of extreme value theory.

Figure 11 plots the bidimensional observations in the physical space and in the rank space (using the empirical distribution functions), where the temperature data have been transformed to their negated values: we note that the upper right corner is quite sparsely populated, which means that extremes seem to occur separately, suggesting asymptotic independence.

To confirm it, we plot the histogram of $W$ from the largest $k$ values in figure 12, where we choose $k$ equal to the number of data for which at least one component exceeds its corresponding threshold. This clearly corresponds to a model with asymptotic independence.

Figure 13 plots the mean excess function for the variable $T$ defined within the Ledford \& Tawn test. The tail coefficient $\eta$ is less than 1 , which discards the asymptotic dependence hypothesis.

The empirical versions of the functions $\chi(u)$ and $\bar{\chi}(u)$ give an idea of the form of the dependence structure at extreme levels. Figure 14 shows that $\chi$ is approximately equal to 0 even for quantile levels close to 1 . The point-wise confidence intervals include the value zero, which confirms the earlier finding of asymptotical independence.

Furthermore, we note that $\bar{\chi}$ is negative which means that the variables exhibit some negative association: when one extreme occurs, it slightly tends to prevent the other one from occurring. 
Pickands dependence function

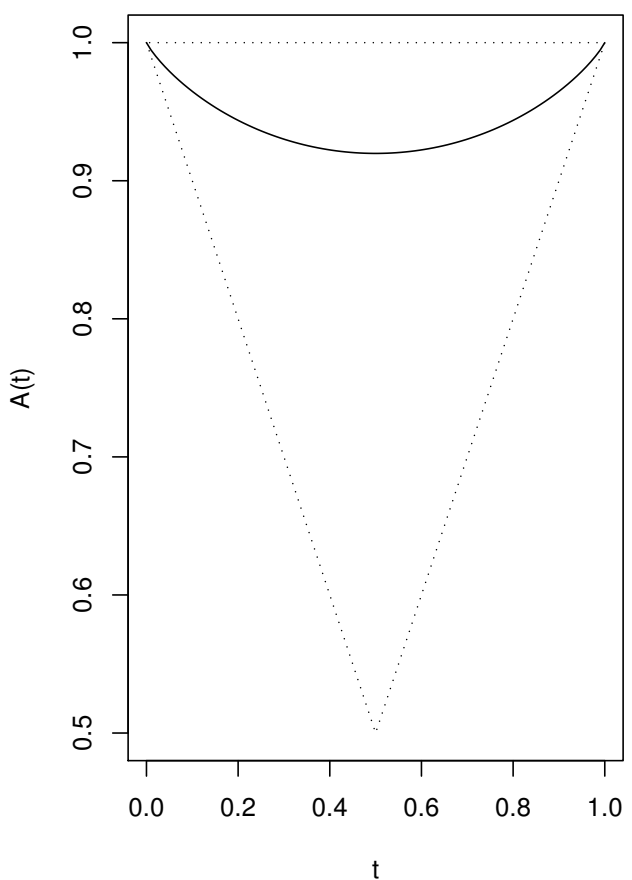

\section{Spectral density}

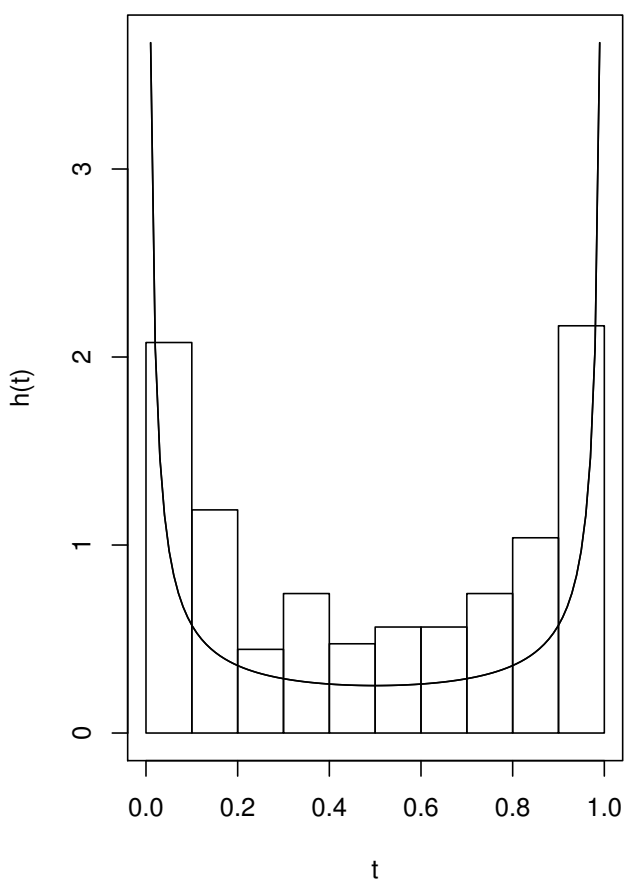

Fig. 7. Goodness of fit of the dependence model: Pickands dependence function and histogram of $W$ for the largest values.

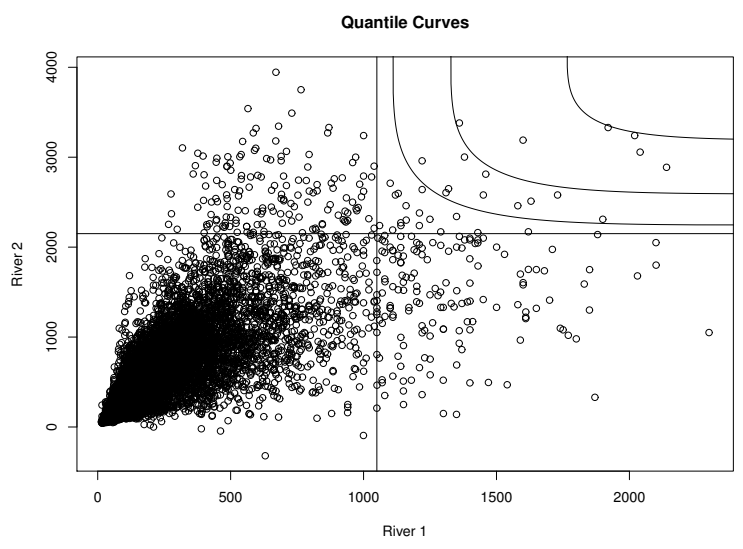

Fig. 8. Some quantile curves of the estimated joint tail model.

This phenomenon is well-known by meteorologists: in winter, the climate of the region where the wind and temperature series are observed is dominated by a westerly flow coming from the ocean. This westerly flow carries with it a succession of low and rather wet and windy conditions, but mild temperatures at the latitude of the studied location. Cold conditions occur when this zonal flow is perturbated, mainly by the installation of an anticyclonic blocking or a ridge. Extremely cold temperatures are thus associated with anticyclonic conditions and rather low to moderate winds.

Actually, the innovative aspect of this study is to bring statistical proof of this feature. Above all, it allows to give a bracketing interval of the probability of the joint occurrence of extremes, as developed in the next section. 


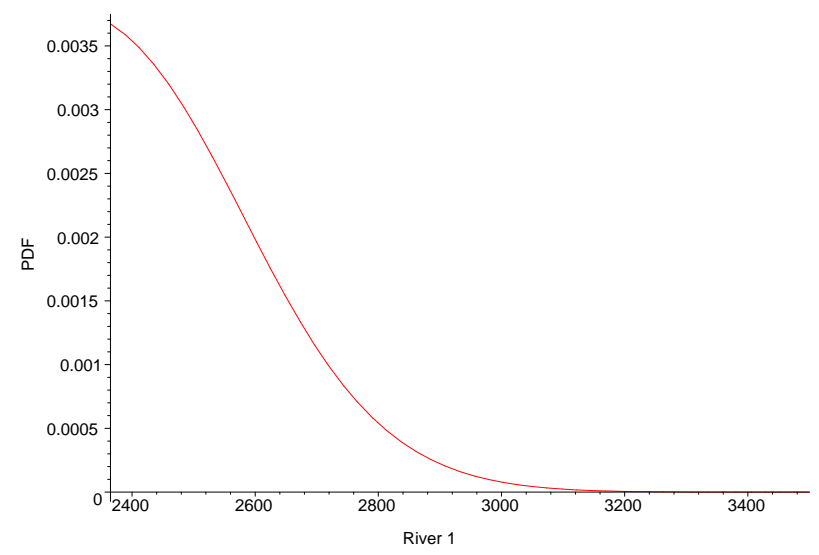

Fig. 9. Probability density function of the first river's discharges when the second river discharge is fixed to its 100-year return level.

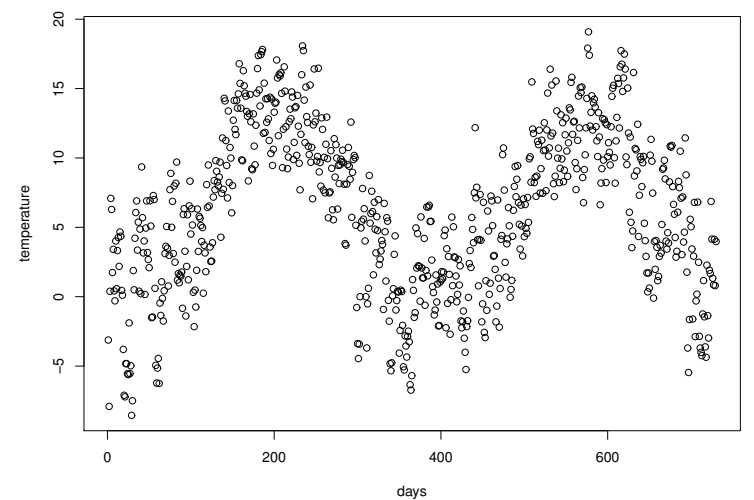

Fig. 10. Air temperature data recorded daily: focus on the period from 01/01/1971 to 31/12/1972.

\subsection{Asymptotic independence exploitation}

As a matter of fact, figure 14 enables us to bracket the function $\bar{\chi}(u)$, as we observe that:

$$
\forall u>0.9,-0.5<\bar{\chi}(u)<-0.2
$$

Thanks to relation (26) and considering that the $(1-u)$ tail quantile $t_{1-u}^{o p p}$ of the negated winter temperature variable $T^{o p p}$ corresponds to the $u$ quantile $t_{u}$ of the temperature variable $T$, it entails that:

$$
(1-u)^{4}<\mathbb{P}\left[V>v_{u}, T<t_{1-u}\right]<(1-u)^{2.5}
$$

where $v_{u}$ is the $u$ tail quantile of the winter wind speed variable $V$ and $t_{1-u}$ the $(1-u)$ quantile of the winter air temperature variable $T$. 


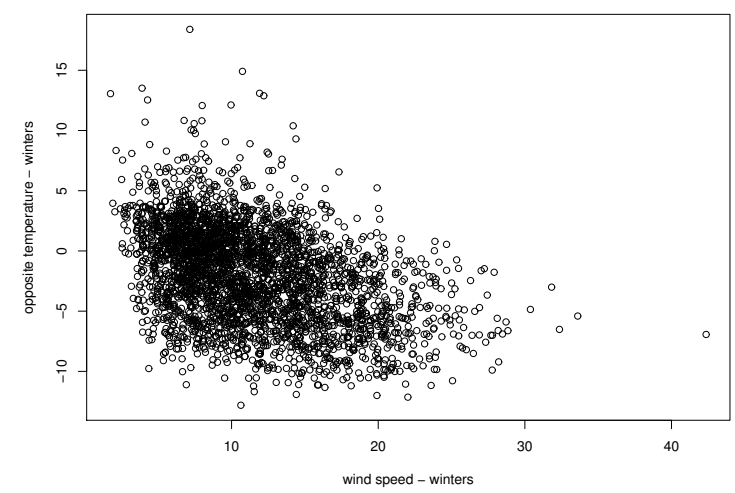

(a)

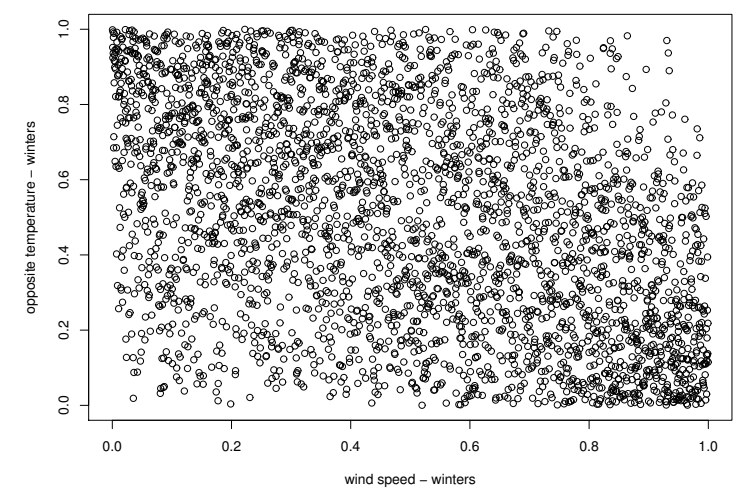

(b)

Fig. 11. Scatterplot of daily joint data (Wind speed, Negated temperature) during the winter seasons of the years 1971 to 2008 : (a) original data; (b) data transformed to uniform distribution.

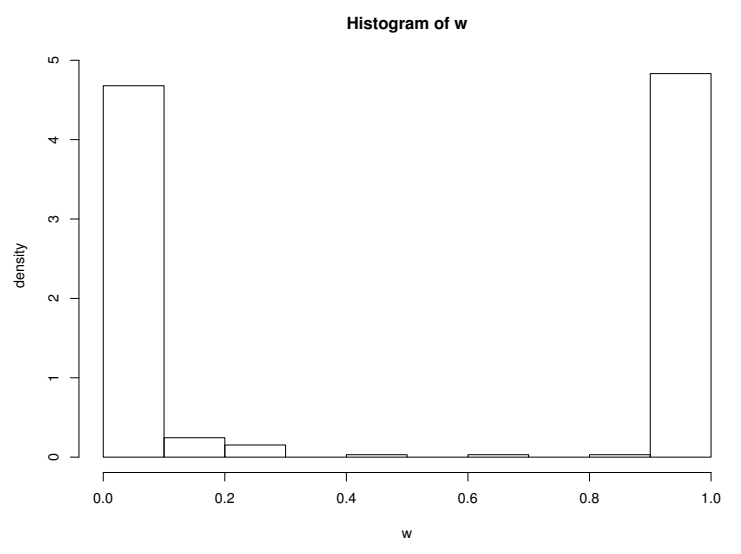

Fig. 12. Histogram of $W$ values in point process analysis of joint data (Negated Temperature, Wind speed).

As the association is negative, the assumption of exact independence leads to an upper bound of the real probability:

$$
\mathbb{P}\left[V>v_{u}, T<t_{1-u}\right]<\mathbb{P}\left[V>v_{u}\right] \mathbb{P}\left[T<t_{1-u}\right]
$$

\section{Summary and Conclusion}

In this paper, we recalled some important results of the analysis of multivariate extreme values. We particularly highlighted the methods that detect asymptotic independence from data and that enable us to quantify the kind of asymptotic independence: positive or negative association. As a matter of fact, such independence makes the class of multivariate extreme value distributions inappropriate to model the tail distribution of data and might lead to underestimated probability of joint occurrence of extreme hazards. We applied the methods on two data sets: one illustrating the asymptotic dependence and the other one illustrating the asymptotic independence. In both cases, we showed how to exploit the results provided by the theory in order to evaluate the probabilistic features of the joint occurrence of two environmental hazards: in the case of asymptotic dependence, we modeled the joint tail distribution function of both random variables, which enabled us to consider some extreme scenarios and evaluate the probabilistic properties of each random variable in their 

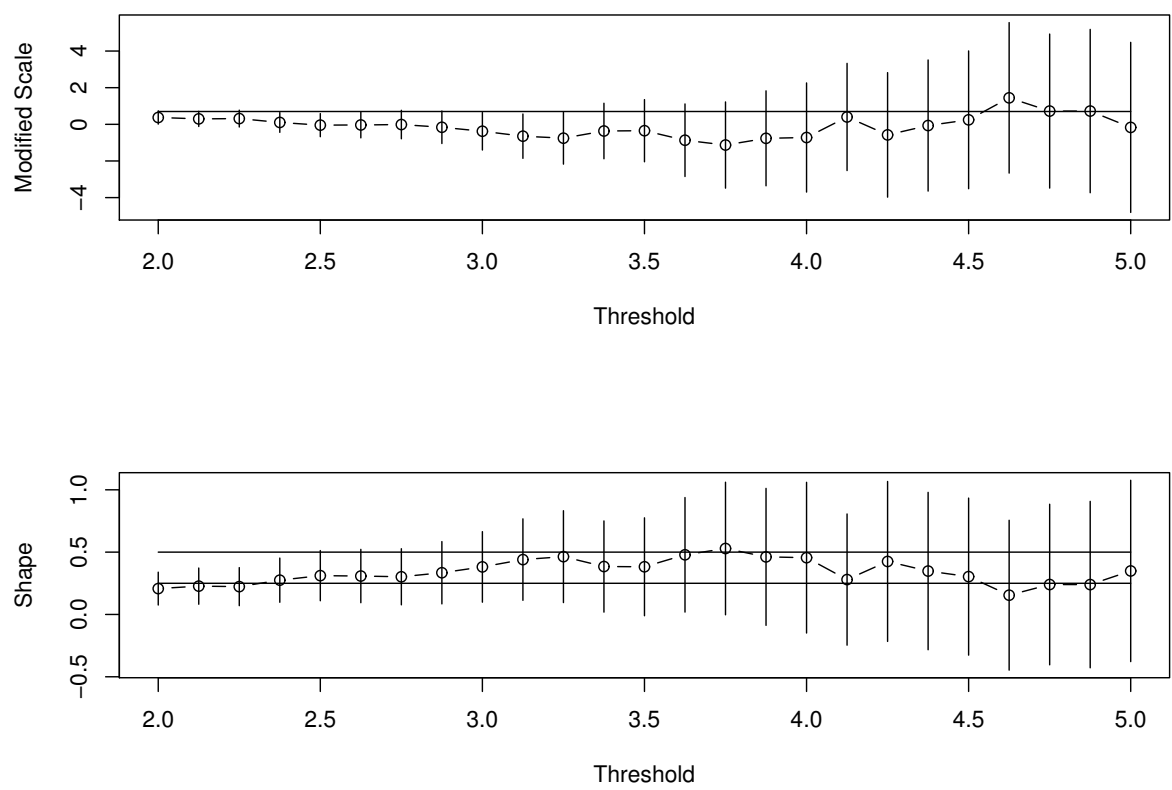

Fig. 13. Estimation of the shape $\eta$ and the modified scale coefficient of the variable $T=\min \left(Z_{1}, Z_{2}\right)$ for several exceedance thresholds for the daily data (Negated Temperature, Wind speed).

Chi Plot

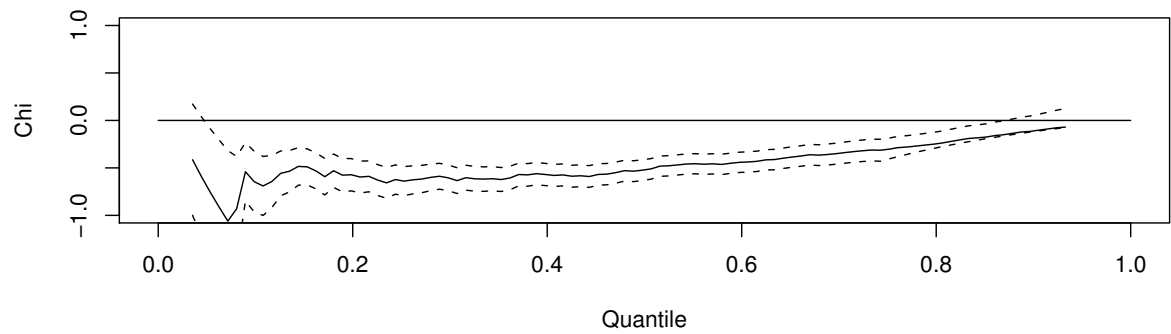

Chi Bar Plot

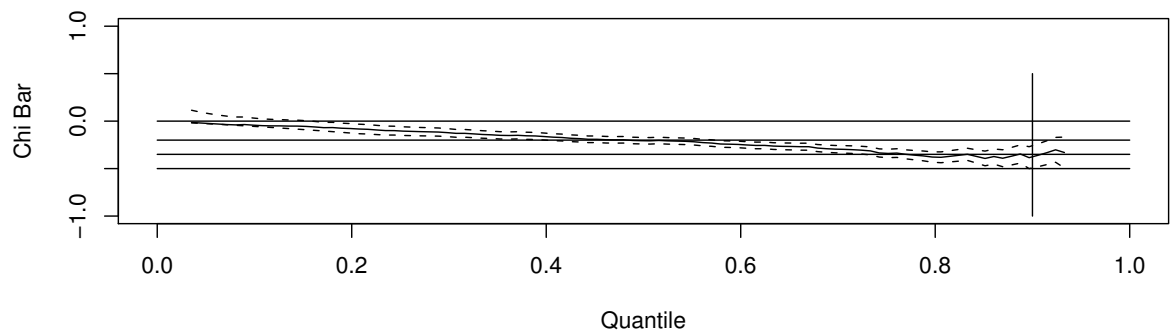

Fig. 14. Empirical estimates of $\chi(u)$ and $\bar{\chi}(u)$ for daily data (Negated Temperature, Wind speed) with $95 \%$ point-wise confidence intervals.

extreme range, while in the case of asymptotic independence, we were able to bracket the probability of joint occurrence of extreme hazards, which we found to be precise enough to design the nearby industrial structures. 


\section{References}

[1] Beirlant, J., Y. Goegebeur, J. Teugels, and J. Segers (2004). Statistics of Extremes, Theory and Applications. John Wiley \& Sons, Ltd., Chichester.

[2] Berman, S. M. (1961/1962). Convergence to bivariate limiting extreme value distributions. Ann. Inst. Statist. Math. 13, 217-223.

[3] Bruun, J. T. and J. A. Tawn (1998). Comparison of approaches for estimating the probability of coastal flooding. J. R. Stat. Soc. Ser. C Appl. Stat. 47(3), 405-423.

[4] Buishand, T. (1989). Statistics of extremes in climatology. Statistica Neerlandica 43(1), 1-30.

[5] Buishand, T. A. (1991). Extreme rainfall estimation by combining data from several sites. Hydrological Sciences Journal 36(4), 345-365.

[6] Capéraà, P. and A.-L. Fougères (2000). Estimation of a bivariate extreme value distribution. Extremes 3(4), 311-329 (2001).

[7] Capéraà, P., A.-L. Fougères, and C. Genest (1997). A nonparametric estimation procedure for bivariate extreme value copulas. Biometrika 84(3), 567-577.

[8] Carter, D. J. T. and P. G. Challenor (1981). Estimating return values of environmental parameters. Q. J. R. Meteorological Soc. 107(451), 259-266.

[9] Chebana, F. and T. B. M. J. Ouarda (2011). Multivariate quantiles in hydrological frequency analysis. Environmetrics 22(1), 63-78.

[10] Coles, S. (2001). An Introduction to Statistical Modeling of Extreme Values. Springer-Verlag London, Ltd., London.

[11] Coles, S. G. and J. A. Tawn (1994). Statistical methods for multivariate extremes: An application to structural design. J. $R$. Stat. Soc. Ser. C Appl. Stat. 43(1), pp. 1-48.

[12] Dales, M. Y. and D. W. Reed (2001). Regional flood and storm hazard assessment. Wallingford, Institute of Hydrology.

[13] de Carvalho, M. and A. Ramos (2012). Bivariate extreme statistics, II. REVSTAT 10(1), 83-107.

[14] de Haan, L. and J. de Ronde (1998). Sea and wind: multivariate extremes at work. Extremes 1(1), 7-45.

[15] de Haan, L. and T. T. Pereira (2006). Spatial extremes: models for the stationary case. Ann. Statist. 34(1), 146-168.

[16] Deheuvels, P. (1991). On the limiting behavior of the Pickands estimator for bivariate extreme-value distributions. Statist. Probab. Lett. 12(5), 429-439.

[17] Di Bernardino, E., V. Maume-Deschamps, and C. Prieur (2013). Estimating a bivariate tail: a copula based approach. J. Multivariate Anal. 119, 81-100.

[18] Dupuis, D. J. and B. L. Jones (2006). Multivariate extreme value theory and its usefulness in understanding risk. N. Am. Actuar. J. 10(4), 1-27.

[19] Durante, F. and G. Salvadori (2010). On the construction of multivariate extreme value models via copulas. Environmetrics 21(2), 143-161.

[20] Embrechts, P., C. Klüppelberg, and T. Mikosch (1997). Modelling Extremal Events for Insurance and Finance. Berlin: Springer.

[21] Falk, M. and R. Michel (2006). Testing for tail independence in extreme value models. Ann. Inst. Statist. Math. 58(2), 261-290.

[22] Galambos, J., J. Lechner, and E. Simiu (Eds.) (1994). Extreme Value Theory and Applications, Dordrecht. Kluwer.

[23] Genest, C. and L.-P. Rivest (1989). A characterization of Gumbel's family of extreme value distributions. Statist. Probab. Lett. 8(3), 207-211.

[24] Gumbel, E. J. (1960). Bivariate exponential distributions. J. Amer. Statist. Assoc. 55, 698-707.

[25] Hall, P. and N. Tajvidi (2000). Distribution and dependence-function estimation for bivariate extreme-value distributions. Bernoulli 6(5), 835-844.

[26] Heffernan, J. E. (2000). A directory of coefficients of tail dependence. Extremes 3(3), 279-290.

[27] Hüsler, J. and D. Li (2009). Testing asymptotic independence in bivariate extremes. J. Statist. Plann. Inference 139(3), 990-998.

[28] Leadbetter, M. R., G. Lindgren, and H. Rootzén (1983). Extremes and Related Properties of Random Sequences and Processes. Springer-Verlag, New York-Berlin.

[29] Ledford, A. W. and J. A. Tawn (1996). Statistics for near independence in multivariate extreme values. Biometrika 83(1), 169-187.

[30] Longin, F. M. (2000). From value at risk to stress testing: The extreme value approach. J. Bank. Financ. 24(7), $1097-1130$.

[31] Maulik, K., S. Resnick, and H. Rootzén (2002). Asymptotic independence and a network traffic model. J. Appl. Probab. 39(4), 671-699.

[32] Mole, N., C. W. Anderson, S. Nadarajah, and C. Wright (1995). A generalized pareto distribution model for high concentrations in short-range atmospheric dispersion. Environmetrics 6(6), 595-606.

[33] Morton, I., J. Bowers, and G. Mould (1997). Estimating return period wave heights and wind speeds using a seasonal point process model. Coastal Engineering 31(1-4), $305-326$.

[34] Nelsen, R. B. (2006). An Introduction to Copulas. New York: Springer. Second edition. 
[35] Parey, S., T. T. H. Hoang, and D. Dacunha-Castelle (2010). Different ways to compute temperature return levels in the climate change context. Environmetrics 21(7-8), 698-718.

[36] Poon, S.-H., M. Rockinger, and J. Tawn (2004). Extreme value dependence in financial markets: Diagnostics, models, and financial implications. Rev. Financ. Stud. 17(2), 581-610.

[37] Resnick, S. and H. Rootzén (2000). Self-similar communication models and very heavy tails. Ann. Appl. Probab. 10(3), 753-778.

[38] Rincón, A. (2012). An index for climate change: a multivariate time series approach. Environmetrics 23(7), 617-622.

[39] Salvadori, G., C. D. Michele, N. Kottegoda, and R. Rosso (2007). Extremes in Nature - An Approach Using Copulas. New York: Springer.

[40] Schlather, M. (2001). Examples for the coefficient of tail dependence and the domain of attraction of a bivariate extreme value distribution. Statist. Probab. Lett. 53(3), 325-329.

[41] Sibuya, M. (1960). Bivariate extreme statistics. I. Ann. Inst. Statist. Math. Tokyo 11, 195-210.

[42] Stărică, C. (1999). Multivariate extremes for models with constant conditional correlations. J. Empir. Financ. 6(5), 515 553.

[43] Tawn, J. A. (1988). Bivariate extreme value theory: models and estimation. Biometrika 75(3), 397-415.

[44] Tawn, J. A. (1990). Modelling multivariate extreme value distributions. Biometrika 77(2), pp. 245-253.

[45] Tsai, Y.-L., D. J. Dupuis, and D. J. Murdoch (2013). A robust test for asymptotic independence of bivariate extremes. Statistics 47(1), 172-183.

[46] Tsai, Y.-L., D. J. Murdoch, and D. J. Dupuis (2011). Influence measures and robust estimators of dependence in multivariate extremes. Extremes 14(4), 343-363.

[47] Weller, G. B., D. S. Cooley, and S. R. Sain (2012). An investigation of the pineapple express phenomenon via bivariate extreme value theory. Environmetrics 23(5), 420-439.

[48] Zhang, D., M. T. Wells, and L. Peng (2008). Nonparametric estimation of the dependence function for a multivariate extreme value distribution. J. of Multivariate Anal. 99(4), 577 - 588.

[49] Zhang, Z., Y. Qi, and X. Ma (2011). Asymptotic independence of correlation coefficients with application to testing hypothesis of independence. Electron. J. Statist. 5, 342-372. 